\title{
Performance Evaluation of Contour Based Segmentation Methods for Ultrasound Images
}

\author{
R. J. Hemalatha $\left(\mathbb{D},{ }^{1}\right.$ V. Vijaybaskar, ${ }^{2}$ and T. R. Thamizhvani ${ }^{3}{ }^{3}$ \\ ${ }^{1}$ Department of Biomedical Engineering, Sathyabama Institute of Science and Technology, Chennai, TamilNadu-600, India \\ ${ }^{2}$ Department of Electronica and Telecommunication, Sathyabama Institute of Science and Technology, Chennai, TamilNadu-600, India \\ ${ }^{3}$ Department of Biomedical Engineering, Vels Institute of Science, Technology and Advanced Studies, Chennai, \\ TamilNadu-600117, India
}

Correspondence should be addressed to R. J. Hemalatha; rjhemalatha@gmail.com

Received 26 May 2018; Revised 9 August 2018; Accepted 30 August 2018; Published 16 September 2018

Academic Editor: Huiyu Zhou

Copyright (c) 2018 R. J. Hemalatha et al. This is an open access article distributed under the Creative Commons Attribution License, which permits unrestricted use, distribution, and reproduction in any medium, provided the original work is properly cited.

\begin{abstract}
Active contour methods are widely used for medical image segmentation. Using level set algorithms the applications of active contour methods have become flexible and convenient. This paper describes the evaluation of the performance of the active contour models using performance metrics and statistical analysis. We have implemented five different methods for segmenting the synovial region in arthritis affected ultrasound image. A comparative analysis between the methods of segmentation was performed and the best segmentation method was identified using similarity criteria, standard error, and F-test. For further analysis, classification of the segmentation techniques using support vector machine (SVM) classifier is performed to determine the absolute method for synovial region detection. With these results, localized region based active contour named Lankton method is defined to be the best segmentation method.
\end{abstract}

\section{Introduction}

Musculoskeletal disorder (MSD), an epidemic disease, is now a significant health problem in emerging and most developing countries in the world. MSD remains the most prevalent disease in society due to its impact on mobility, ability to work, and life style. Arthritis is one of the prevalent MSD among all the age groups of people [1]. The most affected portion is the joint region. At relatively early stage of the disease the synovial membrane around the joints used to inflame and leads to degeneration of the joints. The main system to visualize the joint state is through ultra sound diagnosis (USD). The USD is less expensive and available with all the clinicians $[2,3]$. The USD images represent different tissue regions with variations of gray shades. These images are further processed to segment the synovial region and to analyze the disease condition and further progression of the disease.

One of the essential tasks in image analysis is image segmentation. Segmentation is to extract objects from the images by dividing the image into set of regions with different properties. Segmentation plays an important role in automatic object recognition or pattern identification process to identify pathologies and medical diagnosis $[4,5]$. The most challenging task is to extract the contour and boundaries of the desired region for dynamic analysis of anatomical structures. One of the most robust segmentation methods for medical images is active contour method (ACM).The method implies curve evolution to detect the region of interest in a given image [6-8]. The segmentation process is based on edge and region based approach. The geometric active contour model proposed by Caselles and Malladi et al. is an edge based approach which is based on evolution of curves and geometric flows [9]. Chan and Vese have proposed edge less active contour model which is one of the most wellknown region based method. Bernard et al. have proposed a parameterized active contour method. More recently Li et al. and Lankton proposed a method which utilizes local region information for segmentation [9-14].

In this paper we have applied different segmentation methods to arthritis affected finger joint ultrasound images to segment the synovial region. The efficiency of these 
methods is analyzed using performance analysis metrics and statistical analysis method. For performance analysis metrics we have used Dice coefficient and Hausdorff coefficient and, for statistical analysis method standard error, F-test were used. At the end classification is used to define the absolute active contour method for segmentation of synovial region by training and analyzing the performance metrics and statistical values. The rest of the paper is organized as follows Section 2. The Methods and Materials, Section 3 the results and discussion, and Section 4 the conclusion.

\section{Methods and Materials}

In medical imaging, the segmentation of regions with specific parameters is carried out with the help of active contour models. Because these models develop a contour around the target object and segregate it from the image, the segmented image possesses only the required information of the target object [15]. The level set segmentation methods like Caselles, Chan-Vese, Bernard, Li, and Lankton are applied on arthritis affected finger joint images obtained from the MEDUSA database http://medusa.aei.polsl.pl.[1618]. Further using performance analysis metrics like dice coefficient and Hausdroff distance and statistical analysis metrics like standard error and F-test describes the significant difference between the techniques used for segmentation. Classification using SVM defines the best suited method for synovial region segmentation.

MEDUSA is a standardized and authorized database which consists of finger joint images of different grades (grade 0 , grade 1 , grade 2 , and grade 3 ) of synovitis. Various studies related to arthritis and synovitis are performed using this database.

2.1. Caselles. Caselles is geodesic based active contour methods which largely depend on the level set functions that describe the specific regions in the image for segmentation. Contours are described based on the geometric flow of curve and detection of objects in the image [11]. This type of contour model modifies the curve in the plane by moving the points of the curve perpendicular. The motion of the points is at a speed proportional to the curvature of the region in the image. By adding an area of minimizing region (balloon force), propagation of contour occurs internally by minimization of the interior energy given by

$$
\begin{aligned}
& \mathrm{E}(\mathrm{C})=\int g(I(c(P)))\left\|C^{\prime}(P)\right\| d P \\
& g(I)=\frac{1}{1+\|\nabla(G * I)\|^{2}}
\end{aligned}
$$

I is image intensity, G is Gaussian Filter of unity variance, and $\mathrm{C}$ is derived parametric curve to regions with high gradient where set level function is executed as a signed distance function $(\mathrm{P}=\emptyset(\mathrm{x}))$

Contour models use the energy forces for geometric flow curve description. Geometric contours can be obtained based on regions and edges in the curvature of the image [12].
2.2. Chan-Vese. Chan-Vese is a region based method which segments an image into two homogeneous regions. The method utilizes energy minimization technique defined by weighted values corresponding to the average value of sum of intensity difference from outside and inside the segmented region $[9,10]$. Contours are based on either the variance inside and outside contour or the squared difference between average intensities inside and outside the contours along with the total contour length. This contour model helps to determine different image properties, not only edges, and it also includes regions based on texture and other geometrical features. Energy defines the entire region of interest from the image.

The total energy of the model is given in

$$
\begin{aligned}
& \mathrm{E}_{\emptyset}=\mu \int_{\Omega} \delta(\varphi(\mathrm{a}))|\nabla \varphi(\mathrm{a})| \mathrm{da}+\mathrm{v} \int_{\Omega} \mathrm{H}(\varphi(a)) \mathrm{da} \\
& +\lambda_{1} \int_{\Omega} \mathrm{H}(\varphi(\mathrm{a}))\left|\mathrm{f}-\mathrm{C}_{1}\right| \\
& +\lambda_{2} \int_{\Omega}\left(1-\mathrm{H}(\varphi(\mathrm{a}))\left|\mathrm{f}-\mathrm{C}_{2}\right| \mathrm{da}\right.
\end{aligned}
$$

2.3. Bernard. Bernard method utilizes B-spline coefficients as energy minimization function. These utilize parameterized active contour method [12]. Spline coefficients define the contour models for the pixels of interest. The energy based functions inside and outside is described with these coefficients. Contour models describe the entire structures with inflation force that can overpower forces from weak edges, amplifying the issue with localization of initial guess. To speed up the process a linear combination of B-spline basis functions is used and given in

$$
\begin{aligned}
\mathrm{E}_{\emptyset}= & \int_{\Omega} \mathrm{F}(\mathrm{I}(\mathrm{a}), \emptyset(\mathrm{a})) \mathrm{dx} \\
\mathrm{F}(\mathrm{I}(\mathrm{a}), \emptyset(\mathrm{a}))= & (\mathrm{I}(\mathrm{a})-v)^{2} \mathrm{H}(\emptyset(\mathrm{a})) \\
& +(\mathrm{I}(a)-\mu)^{2}(\mathrm{I}-\mathrm{H}(\emptyset(\mathrm{a}))) .
\end{aligned}
$$

$\Phi($ a) is linear combination of B-spline basis functions.

2.4. Chumming $L i$. In order to separate the region into two homogenous regions this method utilizes using local neighbourhood statistics for each pixel given in (6). It uses local region information for segmentation [13]. The energy function of the region based active contour model is range of region based domain kernel function. By minimizing the 
energy function, the region of elements of the target could be determined in images with contours.

$$
\begin{aligned}
\mathrm{E}_{\emptyset} & =\lambda_{1} \iint \mathrm{K}_{\sigma}(\mathrm{a}-\mathrm{b})\left|\mathrm{I}(\mathrm{b})-\mathrm{f}_{1}(\mathrm{a})\right|^{2} \mathrm{H}(\emptyset(\mathrm{a})) \mathrm{db} \mathrm{da} \\
& +\lambda_{2} \iint \mathrm{K}_{\sigma}(\mathrm{a}-\mathrm{b})\left|\mathrm{I}(\mathrm{b})-\mathrm{f}_{2}(\mathrm{a})\right|^{2} \\
& \cdot(1-\mathrm{H}(\emptyset(\mathrm{a}))) \mathrm{db} \mathrm{da}+v \int \delta(\emptyset(\mathrm{a})) \\
\cdot & \|\nabla \emptyset(\mathrm{a})\| \mathrm{da}+\mu \int \frac{1}{2}(\|\nabla \emptyset(\mathrm{a})\|-1)^{2} \mathrm{da}
\end{aligned}
$$

$$
\begin{aligned}
& \mathrm{I}(\mathrm{a}) \text { : pixel intensity at } \mathrm{x} \\
& \mathrm{H} \text { : heavy side function } \\
& \mathrm{K}_{\sigma} \text { : Gaussian Kernel. }
\end{aligned}
$$

$$
\mathrm{K}_{\sigma}(\mu)=\frac{1}{(2 \pi)^{\pi / 2} \sigma^{\pi}} e^{-\|\mu\|^{2} / 2 \sigma^{2}}
$$

2.5. Lankton. Lankton is a region based active contour method which segments non homogeneous objects. This method utilizes localizing region based energy which segments the region based on local information. It is not suitable for unsupervised image segmentation as it requires appropriate curve initialization [14]. These models form contour boundaries with energy forces required for the particular region of interest. The energy inside and outside depends on the local region pixels of the image that describes the required region. The energy equation is illustrated in

$$
\begin{aligned}
\mathrm{E}_{\emptyset} & =\int_{\Omega} \delta(\emptyset(\mathrm{a})) \int_{\Omega} \mathrm{F}(\mathrm{I}(\mathrm{a}), \emptyset(\mathrm{b})), \mathrm{B}(\mathrm{a}, \mathrm{b}) \mathrm{db} \mathrm{da} \\
& +\lambda \int_{\Omega}\|\nabla \emptyset(\mathrm{a})\| \delta(\emptyset(\mathrm{a})) \mathrm{da}
\end{aligned}
$$

\section{$\delta$ is Dirac function}

$\mathrm{B}$ is Ball of radius $\mathrm{r}$ centered at point $\mathrm{x}$

2.6. Performance Evaluation Metrics. The segmentation methods are qualitatively and quantitatively assessed and compared with each other based on three kinds of criteria. Based on this the best suited algorithm is chosen for particular applications.

Visual criteria: The segmented region using the active contour methods is compared with the annotated images by expert radiologist as reference image. The segmented region obtained from level set function methods is compared with reference image.

Computation time: The time taken for each algorithm to segment the region represents the speed of the algorithm. The speed of the respective algorithm is compared.

Similarity criteria: This criterion measures the similarity between the reference and segmented image. The quality of the segmented image is measured by calculating the Dice coefficient, Hausdorff distance, and PSNR
Dice coefficient compares the segmented region with the reference region from the annotated image and provides the dice coefficient values ranging between 0 and 1 . If it is 1 the segmented region is more similar and it is different when it is $0[19]$.

$$
\text { Dice }=\frac{2(\mathrm{~A} \cap \mathrm{B})}{\mathrm{A}+\mathrm{B}}
$$

Hausdroff distance is a metric to measure dissimilarity between two point sets. Distance transform is used to compute the HD in an image. This is used to control the progress of level set based algorithms and to evaluate the quality of the clusters [20].

$$
D_{1}(A, B)=\max _{x \in A}\left(\min _{y \in B}(\|x-y\|)\right)
$$

2.7. Statistical Analysis. The features like mean, variance, and standard errors are calculated for the segmentation methods. Among the five segmentation methods the best suited method was identified using statistical analysis.

Mean: The mean value is termed as average value which is computed by taking sum of all perceived outcomes divided by overall number of gray levels. The following shows mathematical expression for mean represented as $\bar{x}$ :

$$
\text { Mean, } \bar{x}=\frac{1}{n} \sum_{i=1}^{n} x
$$

where $\mathrm{n}$ is sample size and $\mathrm{x}$ is observed value.

Variance is study of deviation of actual value versus predicted value. The deviation from actual and predicted indicates the performance of the methods used.

Standard error is defined as the measure of prediction's accuracy. Estimated standard error is related to sum of squared deviations of prediction (that is sum of squares error), described

$$
\sigma_{e s t}=\sqrt{\frac{\sum\left(Y-Y^{\prime}\right)^{2}}{N}}
$$

$\sigma_{\text {est }}$ is the standard error of the estimate, $\mathrm{Y}$ is an actual range, $\mathrm{Y}^{\prime}$ is a predicted range, and $\mathrm{N}$ is the number of pairs of scores. $\sum\left(Y-Y^{\prime}\right)^{2}$ is the sum of squared differences between the actual scores and the predicted scores.

2.8. Classification. Classification is a process to describe the effective type or class based on the features derived from the region of interest. Support vector machines (SVM) are machine learning models. SVM is the representation of observations as points that maps to form separate divisions and a clear boundary factor defined as decision boundary. Multiclass support vector machine classifies the types based on the kernel models. Multiclass support vector machine is used to illustrate the appropriate type of active contour technique for the segmentation of the synovial region. 
ORIGINAL IMAGE (ANNOTATED)
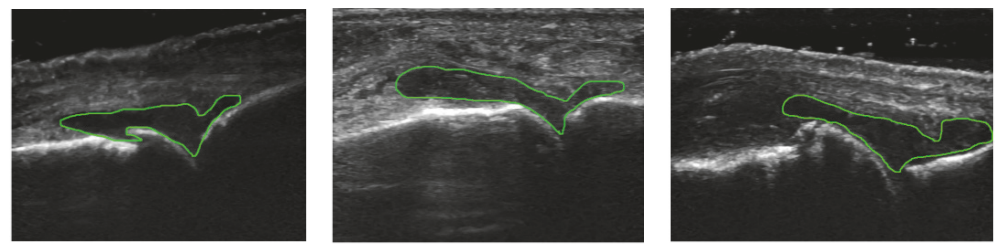

CASELLES
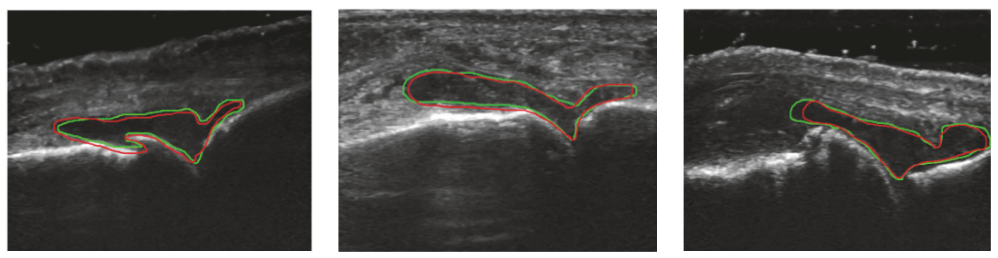

CHAN-VESE
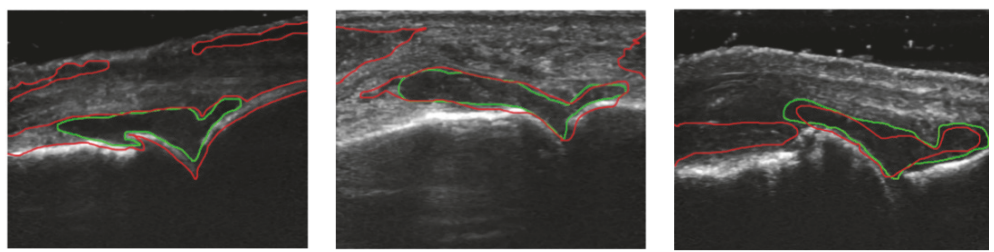

CHUMMING LI
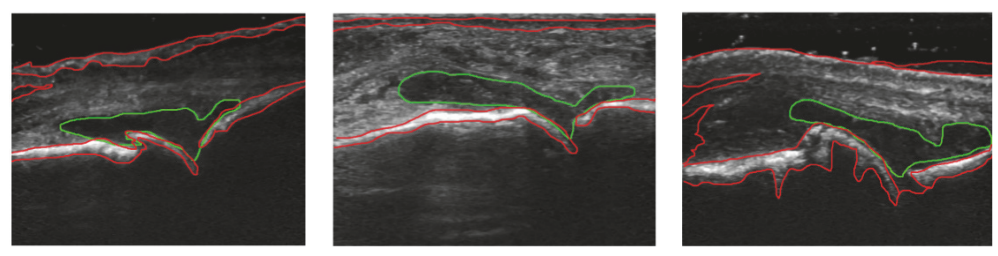

LANKTON
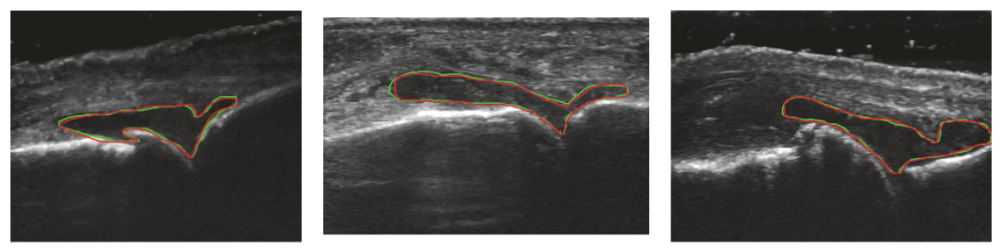

BERNARD
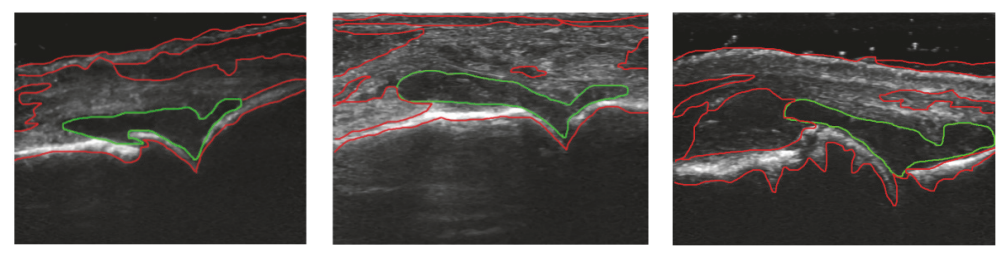

FIGURE 1: Different types of active contour segmentation techniques.

\section{Results}

In this method, different types of active contour segmentation techniques are used for the detection of synovial region. The segmentation methods were evaluated using performance metrics and statistical analysis. Ultrasound images from the database are used for the identification of synovial region. Fifty images of different grades are considered for segmentation of the synovial region from the database. Different active contour segmentation techniques are used to segment the synovial regions. Visual changes in the segmentation process are illustrated through the images in Figure 1.

In this figure, the annotated image is defined with green colour and the synovial region defined by the five different types of segmentation is displayed in red colour. The 

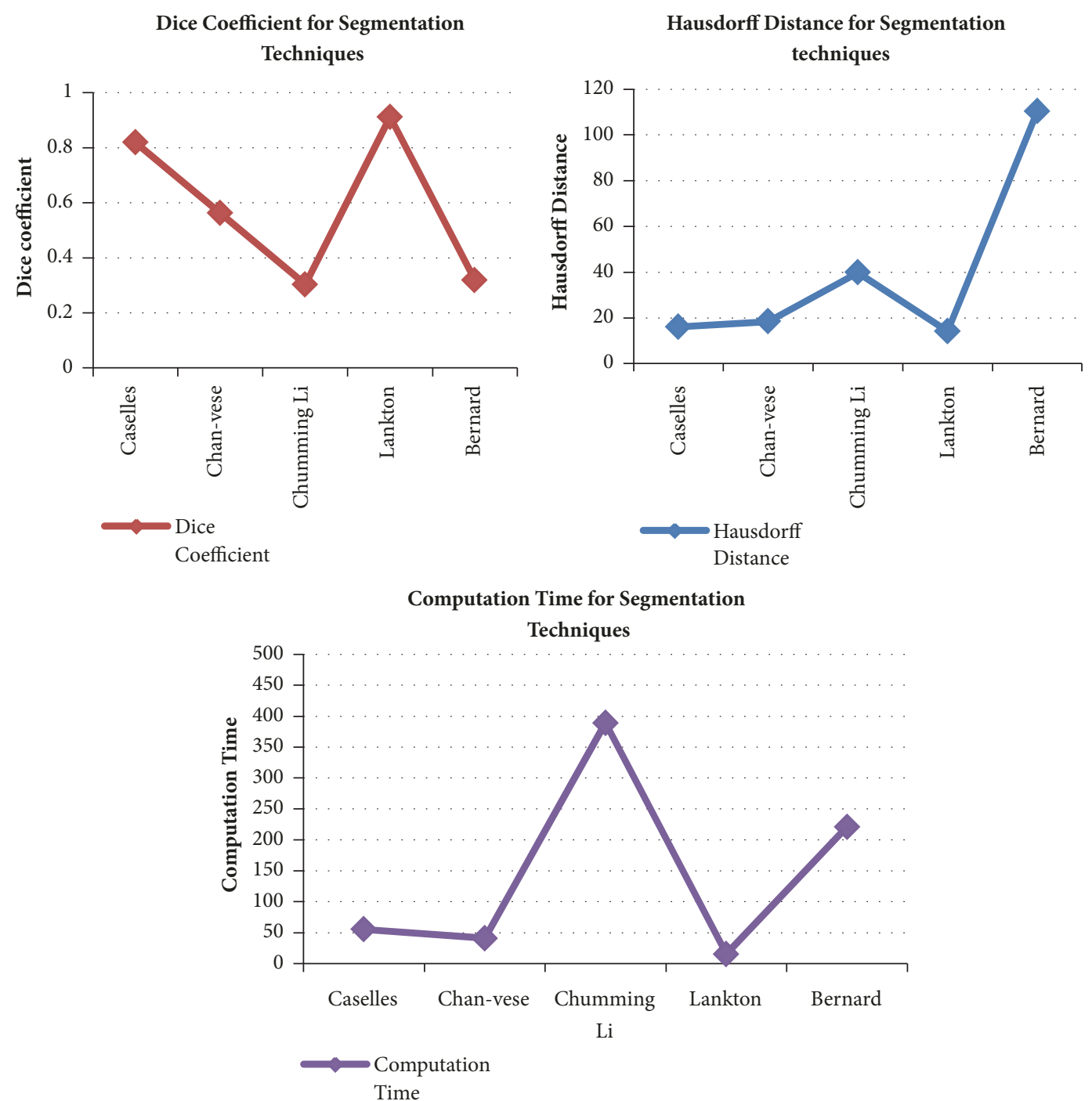

FIGURE 2: Graphical representation of the performance metrics of five different active contour techniques.

TABLE 1: Performance metrics value for several segmentation methods.

\begin{tabular}{lccc}
\hline $\begin{array}{l}\text { Segmentation } \\
\text { methods }\end{array}$ & $\begin{array}{c}\text { Dice } \\
\text { Coefficient }\end{array}$ & $\begin{array}{c}\text { Hausdorff } \\
\text { Distance }\end{array}$ & $\begin{array}{c}\text { Computation } \\
\text { Time }\end{array}$ \\
\hline Caselles & 0.823 & 16.136 & 40.608 \\
Chan-Vese & 0.562 & 18.419 & 55.109 \\
Chumming Li & 0.303 & 39.841 & 388.815 \\
Lankton & 0.879 & 14.814 & 13.416 \\
Bernard & 0.204 & 88.601 & 402.057 \\
\hline
\end{tabular}

segmented synovial region, when compared to the annotated images, visually defines the fact that Caselles and Lankton possess similarity. To analyze the absolute segmentation technique, further performance metrics description, statistical analysis, and classification are carried out. The performance analysis metrics like dice coefficient, Hausdorff distance, and computation time values of an image is tabulated in Table 1.
Comparison between each performance metric of the five different active contour techniques is graphically represented in Figure 2. These representations illustrate that the Caselles geodesic active contour and Lankton localized region based active contour methods have slightly similar values. From the dice coefficient values it is shown that Caselles and Lankton are more towards 1 that is the highest range. Hausdroff distance also shows the similarity between the two methods. Computation time in seconds defines the fact that the localized region based active contour Lankton is efficient. To further classify which is the best suitable segmentation technique we perform statistical analysis.

In statistical analysis, the features like standard error, average mean, and average variance values are derived from the similarity index parameters (Dice coefficient and Hausdroff distance) for the exact determination of the segmentation technique. In this Table 2 shows the statistical analysis of the similarity index parameters in performance metrics defining the average mean, standard error, and average variance values. 
TABLE 2: Statistical values of performance metrics for segmentation techniques.

\begin{tabular}{lcccccc}
\hline \multirow{2}{*}{ Segmentation methods } & \multicolumn{2}{c}{ Average Mean } & \multicolumn{2}{c}{ Standard Error } & \multicolumn{2}{c}{ Average Variance } \\
& Dice coefficient & Hausdorff distance & Dice coefficient & Hausdorff distance & Dice coefficient & Hausdorff distance \\
\hline Caselles & $0.809 \pm 0.060$ & $30 \pm 15.60$ & $0.809 \pm 0.0837$ & $30 \pm 11.18$ & $0.809 \pm 0.0451$ & $30 \pm 103.32$ \\
Chan-vese & $0.579 \pm 0.150$ & $90.9 \pm 25.40$ & $0.579 \pm 0.1404$ & $90.9 \pm 56.82$ & $0.579 \pm 0.0233$ & $90.9 \pm 46.10$ \\
Chumming Li & $0.306 \pm 0.050$ & $235.17 \pm 100$ & $0.306 \pm 0.1829$ & $235.17 \pm 6.570$ & $0.306 \pm 0.0520$ & $235.17 \pm 862$ \\
Lankton & $0.873 \pm 0.005$ & $18.7 \pm 0.010$ & $0.873 \pm 0.0858$ & $18.7 \pm 13.31$ & $0.873 \pm 0.0403$ & $18.7 \pm 37.92$ \\
Bernard & $0.158 \pm 0.160$ & $284.4 \pm 200$ & $0.158 \pm 0.0907$ & $284.4 \pm 11.18$ & $0.158 \pm 0.0081$ & $284.4 \pm 489$ \\
\hline
\end{tabular}

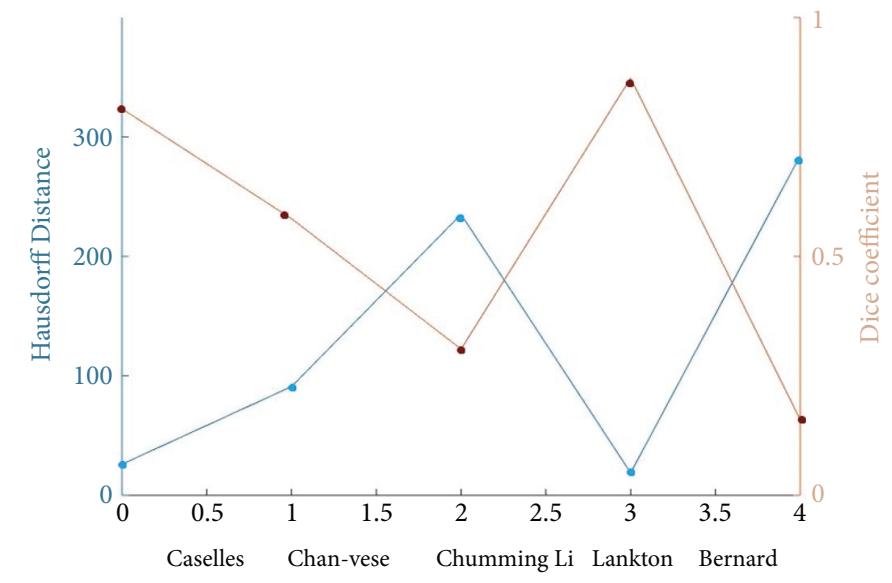

FIGURE 3: Graphical representation of mean average values of similarity index parameters.

Mean average value defines significant variations among all the types of active contour techniques used for the process of segmentation of the synovial region. But the values possess slight similarity between the two active contour methods. The slight deviation in the synovial segmentation between the two methods is illustrated in Figure 3 which represents the mean values of the similarity index parameters of the five active contour methods.

The standard error measurement describes the accuracy of the predicted value. The difference in standard error between Caselles and Lankton active contours is 0.0021 , which is really insignificant. So there is a little more similarity among the geodesic and localized region based active contours.

Average variance is derived to find out the significant difference between the methods. The difference can be defined appropriately using F-test which is performed over the variance value. The F-test results shows that the Caselles geodesic active contour and Lankton localized region based active contour method are likely significant which shows that the region segmented using these methods is not similar. The result of the F-test of the geodesic active contour Caselles and localized region based active contour Lankton is shown in Table 3.

As a result of statistical analysis, Caselles and Lankton have slightly significant difference and prove to possess dissimilarity in the process of segmentation of synovial region. For further determination of the exact segmentation technique, the performance metrics and statistical features obtained from the region undergo classification process. In this process, support vector machine (SVM) classifier is used for training and identification of the significant segmentation technique for synovial region detection. Localized region based active contour Lankton is described as more significant with the help of the results of classification. These results are defined with confusion matrix and scatter plot of the trained features of the synovial region from the ultrasound images. In Figure 4, the confusion matrix is defined with accuracy rates of each category of segmentation. With these results, Lankton is determined as the significant nature of the method in detection of synovial region. Features extracted from the synovial region possess significant variations and localized region based active contour is described with the accuracy of the confusion matrix based on the true positive and false negative rates.

From these results, Localized region based active contour is more efficient method of active contours for synovial region segmentation by training and classifying the features like performance metrics and statistical values. These features define the appropriate classification of the region which is performed with this Lankton active contour.

\section{Conclusion}

In recent days arthritis has become a significant health problem. Early diagnosis and treatment help the patients to lead normal life. A method was presented to evaluate the active contour segmentation algorithms to segment the synovial region from arthritis affected finger ultrasound image. Performance analysis metrics like Dice coefficient and 
TABLE 3: F-Test for Variances of Caselles and Lankton Method.

\begin{tabular}{|c|c|c|c|c|c|}
\hline \multicolumn{3}{|c|}{ F-Test Two-Sample for Dice coefficient Variances } & \multicolumn{3}{|c|}{ F-Test Two-Sample for Hausdorff distance Variances } \\
\hline Mean & 0.845719 & 0.829609 & Mean & 19.47198 & 27.57708 \\
\hline Variance & 0.04031 & 0.045104 & Variance & 37.91567 & 103.3178 \\
\hline Observations & 8 & 8 & Observations & 8 & 8 \\
\hline df & 7 & 7 & df & 7 & 7 \\
\hline $\mathrm{F}$ & 0.893709 & & $\mathrm{~F}$ & 0.366981 & \\
\hline $\mathrm{P}(\mathrm{F}<=\mathrm{f})$ one-tail & 0.442978 & & $\mathrm{P}(\mathrm{F}<=\mathrm{f})$ one-tail & 0.104682 & \\
\hline $\begin{array}{l}\text { F Critical } \\
\text { one-tail }\end{array}$ & 0.264058 & & F Critical one-tail & 0.264058 & \\
\hline
\end{tabular}

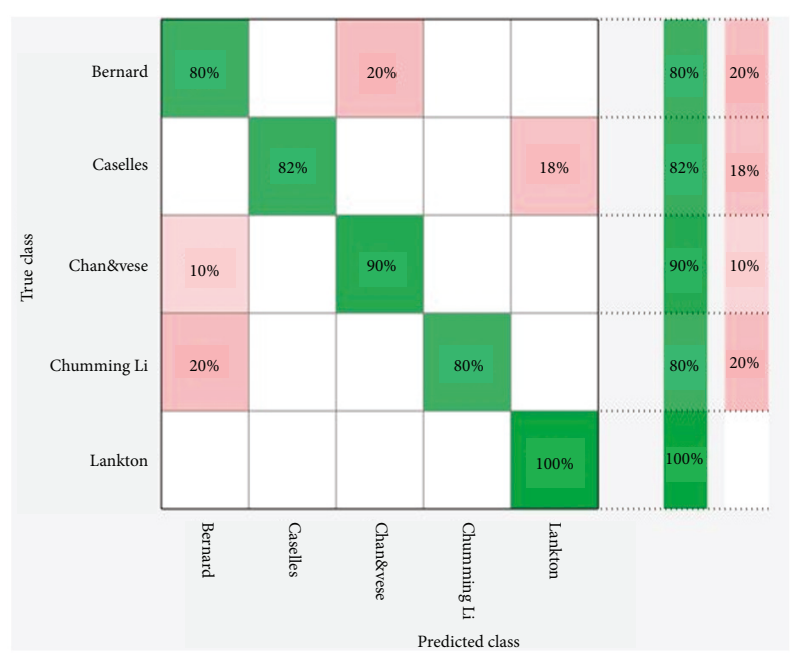

FIGURE 4: Confusion matrix of the classification of active contour segmentation techniques.

Hausdroff distance and statistical analysis metrics like standard error and F-test shows the significant difference between the two segmentation method (Caselles and Lankton) for synovial region. Further classification is performed for the derived features such as performance metrics and statistical values. Higher accuracy is described for Lankton as the result of classification process. Hence the output of the research work shows that Lankton method is the best method for synovial region segmentation from ultrasound images.

\section{Data Availability}

The ultrasound images used to support the findings of this study were supplied by Krystian.Radlak under license agreement from MEDUSA Project and so cannot be made freely available. Requests for access to these data should be made to Krystian.Radlak, krsytian.radlak@polsl.pl.

\section{Additional Points}

The complete research work concentrates on evaluation of contour based segmentation techniques.

\section{Conflicts of Interest}

There are no conflicts of interest among the authors with regard to the proposed methodology and performance evaluation for contour based segmentation techniques for ultrasound images using statistical analysis.

\section{References}

[1] R. Sharma, Ed., Epidemiology of Musculoskeletal Conditions in India, International Journal on Computer Science and Engineering (IJCSE), New Delhi, India, 2012.

[2] A. Bk, J. Segen, K. Wereszczyski, P. Mielnik, M. Fojcik, and M. Kulbacki, "Detection of linear features including bone and skin areas in ultrasound images of joints," PeerJ, vol. 2018, no. 3, 2018.

[3] G. Schett, "Synovitis-an inflammation of joints destroying the bone.”, Swiss Medical Weekly, vol. 142, p. w13692, 2012.

[4] M. Krishnaveni, "Quantitative evaluation of Segmentation algorithms based on level set method for ISL datasets," International Journal on Computer Science and Engineering (IJCSE), vol. 3, pp. 2361-2369, 2011.

[5] D. Barbosa, T. Dietenbeck, J. Schaerer, J. D’Hooge, D. Friboulet, and O. Bernard, "B-spline explicit active surfaces: an efficient framework for real-time 3-D region-based segmentation," IEEE Transactions on Image Processing, vol. 21, no. 1, pp. 241-251, 2012.

[6] D. Reska, C. Boldak, and M. Kretowski, "A Texture-Based Energy for Active Contour Image Segmentation," in Image Processing Communications Challenges, Advances in Intelligent Systems and Computing, R. Choras, Ed., vol. 313, Springer, Cham, 2015.

[7] M. Airouche, L. Bentabet, and M. Zelmat, "Image Segmentation Using Active Contour Model and Level Set Method Applied to Detect Oil Spills," in Proceedings of the In Proceedings of the World Congress on Engineering, vol. I, pp. 846-850, London, U.K, 2009.

[8] R. Goldenberg, R. Kimmel, E. Rivlin, and M. Rudzsky, "Fast geodesic active contours," IEEE Transactions on Image Processing, vol. 10, no. 10, pp. 1467-1475, 2001.

[9] T. Chan and L. Vese, "An active contour model without edges," in Scale-Space Theories in Computer Vision, vol. 1682 of Lecture Notes in Computer Science, pp. 141-151, Springer, Berlin, Germany, 1999.

[10] P. Getreuer, "Chan-Vese Segmentation," Image Processing On Line, vol. 2, pp. 214-224, 2012.

[11] V. Caselles, F. Catté, T. Coll, and F. Dibos, "A geometric model for active contours in image processing," Numerische Mathematik, vol. 66, no. 1, pp. 1-31, 1993. 
[12] O. Bernard, D. Friboulet, P. Thevenaz, and M. Unser, "Variational B-spline level-set: a linear filtering approach for fast deformable model evolution," IEEE Transactions on Image Processing, vol. 18, no. 6, pp. 1179-1191, 2009.

[13] C. Li, C. Y. Kao, J. C. Gore, and Z. Ding, "Minimization of region-scalable fitting energy for image segmentation," IEEE Transactions on Image Processing, vol. 17, no. 10, pp. 1940-1949, 2008.

[14] S. Lankton and A. Tannenbaum, "Localizing region-based active contours," IEEE Transactions on Image Processing, vol. 17, no. 11, pp. 2029-2039, 2008.

[15] A. Khadidos, V. Sanchez, and C.-T. Li, "Active contours based on weighted gradient vector flow and balloon forces for medical image segmentation," pp. 902-906.

[16] MEDUSA, "Automated assessment of joint synovitis activity from medical ultrasound and power Doppler examinations using image processing and machine learning methods," http://eeagrants.org/project-portal/project/PL12-0015.

[17] K. Radlak, N. Radlak, and B. Smolka, "Automatic detection of bones based on the confidence map for Rheumatoid Arthritis analysis," in Proceedings of the 5th Eccomas Thematic Conference on Computational Vision and Medical Image Processing, VipIMAGE 2015, pp. 215-220, Spain, October 2015.

[18] P. Mielnik, M. Fojcik, J. Segen, and M. Kulbacki, "A Novel Method of Synovitis Stratification in Ultrasound Using Machine Learning Algorithms: Results From Clinical Validation of the MEDUSA Project," Ultrasound in Medicine \& Biology, vol. 44, no. 2, pp. 489-494, 2018.

[19] T. Dietenbeck, M. Alessandrini, D. Friboulet, and O. Bernard, "Creaseg: A free software for the evaluation of image segmentation algorithms based on level-set," in Proceedings of the 2010 17th IEEE International Conference on Image Processing, ICIP 2010, pp. 665-668, Hong Kong, September 2010.

[20] A. A. Taha and A. Hanbury, "An efficient algorithm for calculating the exact hausdorff distance," IEEE Transactions on Pattern Analysis and Machine Intelligence, vol. 37, no. 11, pp. 2153-2163, 2015. 


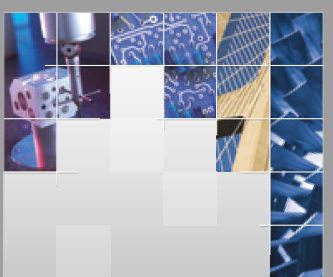

\section{Enfincering}
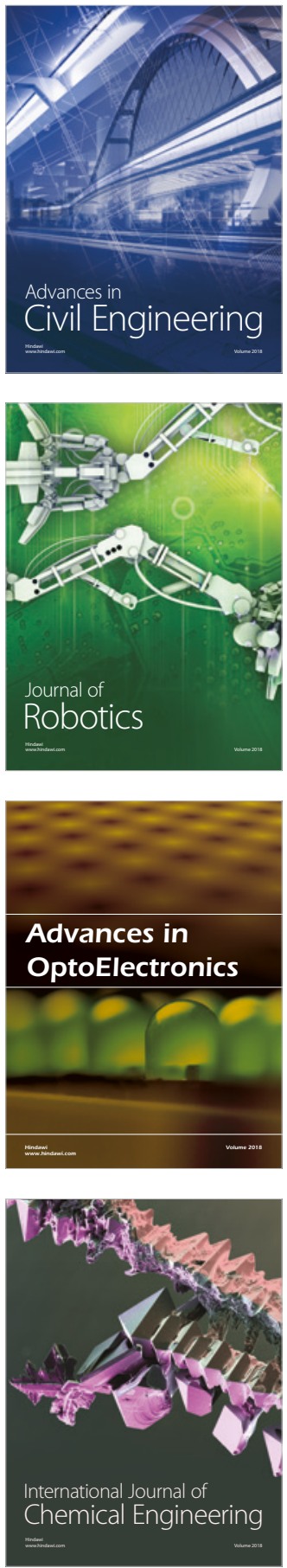

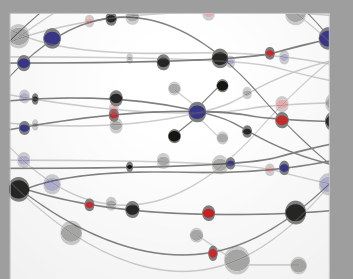

\section{Rotating \\ Machinery}

The Scientific World Journal

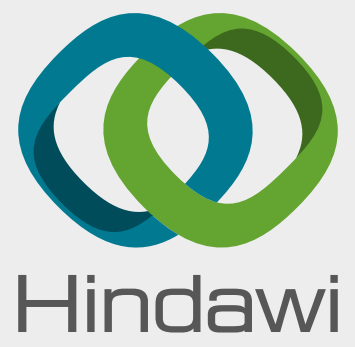

Submit your manuscripts at

www.hindawi.com
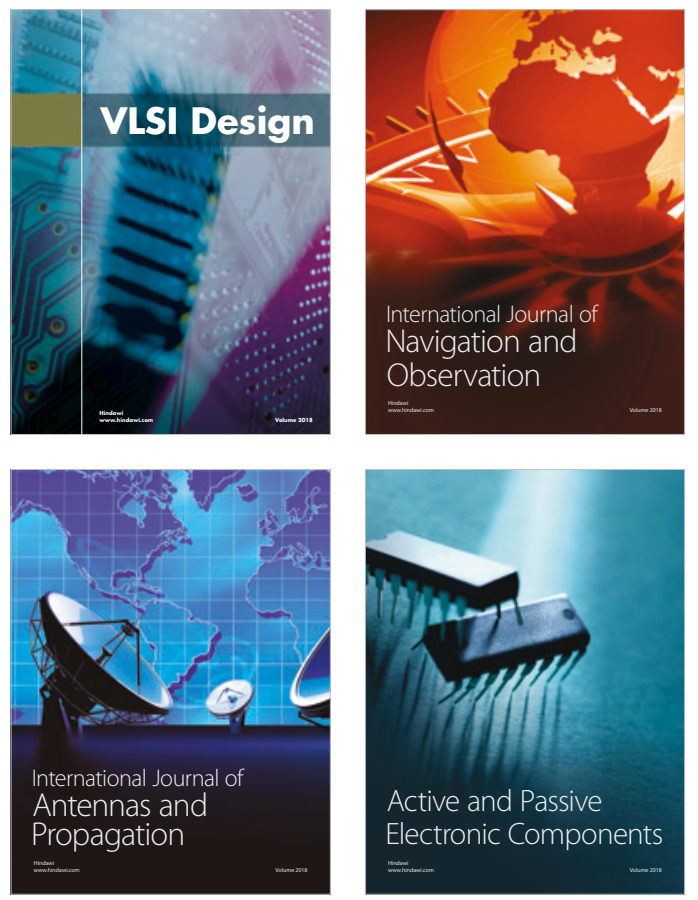
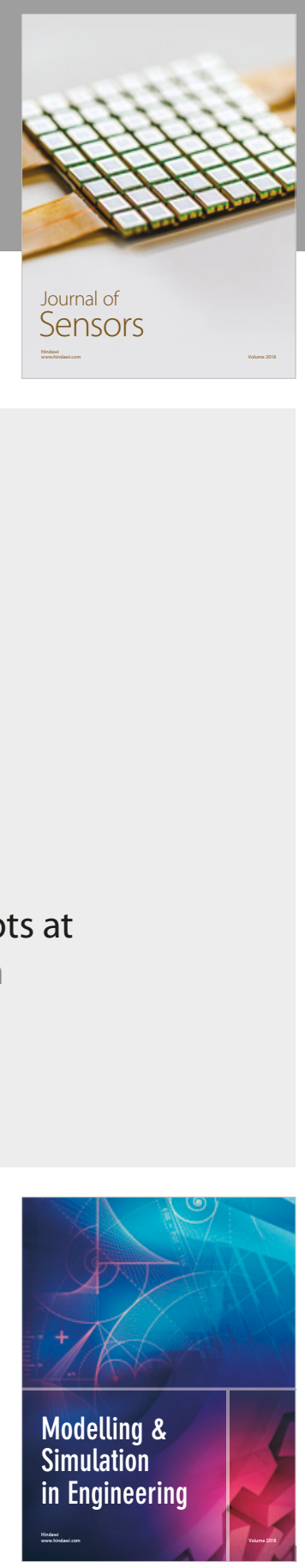

\section{Advances \\ Multimedia}
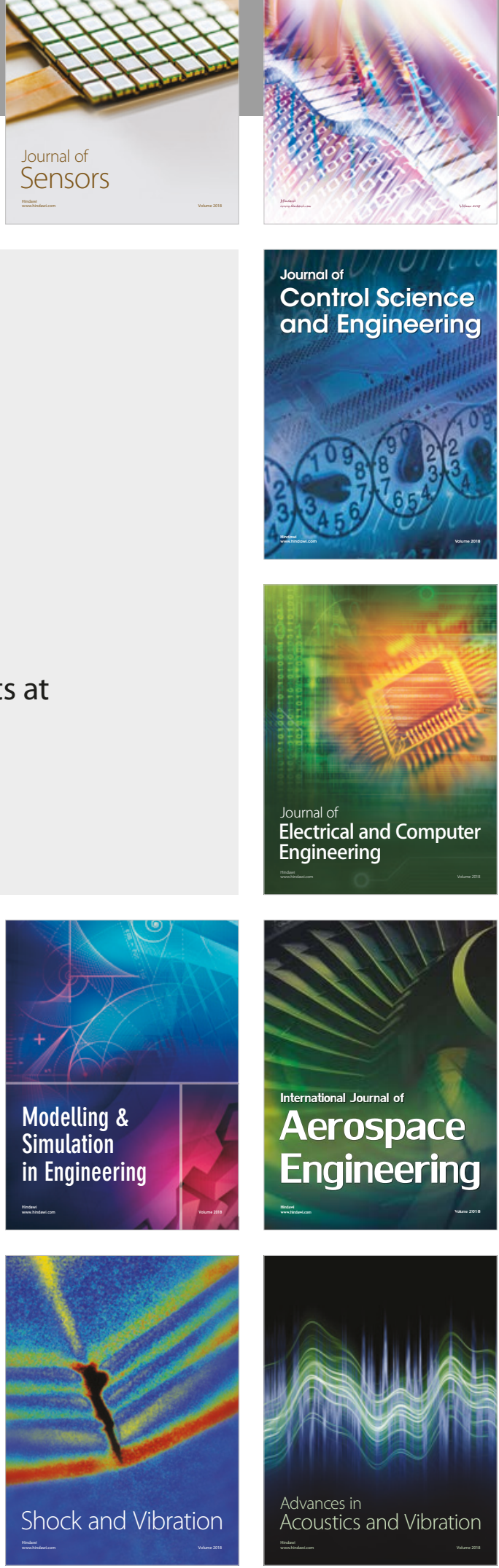